\title{
High frequency of primary cutaneous lymphomas associated with lymphoproliferative disorders of different lineage
}

\author{
Christian Hallermann • Kjell Matthias Kaune • \\ Markus Tiemann • Ekkehard Kunze • \\ Frank Griesinger • Christina Mitteldorf • \\ Hans-Peter Bertsch • Christine Neumann
}

Published online: 21 March 2007

(C) Springer-Verlag 2007

The name of the second author was rendered incorrectly.

The correct version of his name is as shown here.

Acknowledgement Christian Hallermann and Kjell Matthias Kaune contributed equally.

The online version of the original article can be found at http://dx.doi. org/10.1007/s00277-007-0276-8.

C. Hallermann · K. M. Kaune $\cdot$ C. Mitteldorf · H.-P. Bertsch •

C. Neumann

Department of Dermatology, University of Goettingen,

Goettingen, Germany

C. Hallermann $(\bowtie)$

Fachklinik Hornheide,

Dorbaumstrasse 300,

48157 Muenster, Germany

e-mail: christian.hallermann@fachklinik-hornheide.de

M. Tiemann

Department of Haematopathology and Lymph Node Registry,

University Hospitals of Schleswig-Holstein,

Campus Kiel,

Kiel, Germany

E. Kunze

Department of Osteopathology and Haematopathology,

University of Goettingen,

Goettingen, Germany

F. Griesinger

Department of Haematology and Oncology,

University of Goettingen,

Goettingen, Germany 